\title{
Xenopus nerve-muscle cultures: a novel cell-based assay for serological diagnosis and pathological research of myasthenia gravis
}

Zora Chui Kuen Chan, B.Sc. • Marilyn Janice Oentaryo, B.Sc. • Chi Wai Lee, B.Sc., Ph.D.

School of Biomedical Sciences, LKS Faculty of Medicine, The University of Hong Kong, Hong Kong

Keywords: Xenopus, tissue culture, neuromuscular junction, acetylcholine receptor, myasthenia gravis, cell-based assay

Zora Chui Kuen Chan (Email: zoracck@hku.hk)

Marilyn Janice Oentaryo (Email: mjanicee@,hku.hk)

Chi Wai Lee* (Email: chiwai.lee@hku.hk)

School of Biomedical Sciences, LKS Faculty of Medicine, The University of Hong Kong, 21 Sassoon Road, Pokfulam, Hong Kong.

${ }^{*}$ Corresponding author 


\section{Abstract}

\section{Purpose of review:}

At present, the most common diagnostic measures for the autoimmune neuromuscular disease myasthenia gravis (MG) are radioimmunoprecipitation assay (RIPA), enzyme-linked immunosorbent assay (ELISA), and cell-based assay (CBA). Considering the pitfalls of these diagnostic assays, this review describes the advantages of using Xenopus tissue cultures for MG diagnosis and research.

\section{Recent findings:}

Our recent study described a novel CBA involving Xenopus tissue cultures for MG serological diagnosis. Moreover, this CBA can potentially be applied to elucidate the pathogenic mechanisms underlying $\mathrm{AChR}$ endocytosis and degradation, and to develop and validate potential therapeutic strategies for MG.

\section{Summary:}

Although most CBAs are relatively labor intensive, Xenopus CBA is a promising tool for the initial clinical serological diagnosis and for the pathological research of MG. The future studies will be devoted to gain a better understanding of the etiology of MG and to provide a therapeutic intervention for this disease. 


\section{Introduction}

The African clawed frog Xenopus is the leading vertebrate model for the study of gene function in embryonic development for the past $50+$ years $[1,2]$. Using a combination of various techniques, including molecular manipulation, cell lineage analysis, and live-cell imaging, Xenopus offers an ideal platform for studies on the molecular and cellular controls of patterning formation, morphogenesis and organogenesis during embryonic development. Remarkably, the well-established Xenopus primary cultures of nerve and muscle tissues have been used extensively to elucidate the cellular and molecular mechanisms of neuromuscular junction (NMJ) development [3, 4]. Embryonic Xenopus tissue cultures provide several unique advantages, including the feasibility of forming functional NMJs in vitro by coculturing the muscle cells and spinal neurons (Figure 1a, b), over-expression of green fluorescent protein (GFP) fusion proteins or their mutant, and morpholino-mediated knockdown of a specific endogenously expressed protein can be easily performed in early Xenopus embryos. Importantly, many findings originally discovered in Xenopus system have been subsequently validated in the mammalian systems $[5,6]$.

Myasthenia gravis (MG) is an acquired autoimmune disease of NMJ with a prevalence of 200-300 per million populations. MG is heterogeneous in terms of pathophysiology, which is determined by the pathogenic autoantibodies (autoAbs) targeting to synaptic proteins at the NMJs [7•]. Approximately $85 \%$ of generalized MG patients have autoAbs against the acetylcholine receptors (AChRs) in the postsynaptic muscle membrane [8], and about $60 \%$ of the remaining patients have autoAbs against the muscle associated receptor tyrosine kinase (MuSK) and LDL receptor related protein 4 (LRP4) $[9,10]$. In this review paper, we will describe our recently developed assay involving the use of Xenopus muscle or nerve-muscle cultures as an alternative CBA for MG diagnosis. Afterwards, we will highlight the strengths of Xenopus tissue cultures as a research platform to elucidate the pathogenic mechanisms underlying AChR endocytosis in MG patients, and to develop potential therapeutic strategies by manipulating AChR endocytosis and degradation in $\mathrm{MG}$ pathogenesis.

\section{Development of functional NMJs in embryonic Xenopus nerve-muscle cultures}

The NMJ is comprised of the presynaptic motor nerve terminal, the postsynaptic muscle membrane, and the terminal Schwann cell [11]. The basal lamina components at synaptic cleft also contribute a crucial role in synaptic development. The study of NMJ 
development in tissue cultures is traceable back to the landmark discovery by Ross Harrison in 1907, in which he invented the "hanging drop" technique using the combined frog embryonic nerve fragments and lymphoid tissue to prepare explants on sterile coverslips [12]. This denotes the very first observation of nerve tissues that can be cultured, maintained, and grown outside the body. Anderson et al. have further developed the preparation of nervemuscle co-cultures using dissociated tissues from early Xenopus embryos in the late 1970s [13]. They discovered that the formation of NMJs in vitro can be reproducibly observed when the dissociated Xenopus spinal neurons and muscle cells are cultured together, as evidenced by the accumulation of AChRs at nerve-muscle contact sites after the labeling with fluorescent $\alpha$-bungarotoxin ( $\alpha$-BTX) (Figure 1b). Moreover, by making successive observations on the same muscle cells, Anderson and Cohen demonstrated the re-distribution of fluorescently labeled AChRs upon synaptic induction [14]. Nerve-induced redistribution of AChRs is thus believed to be one of the initial steps in neuromuscular synaptogenesis. The development of functional NMJs after the initial establishment of a nerve-muscle contact was further supported by in vitro electrophysiological recordings on Xenopus nerve-muscle co-cultures [15] (Figure 1c). Similar to the NMJs in mammalian systems, the developmental transition of AChR subunit composition from $\gamma$ - to $\varepsilon$-subunit is also detected at Xenopus NMJs [16], reflecting the modulation of AChR channel properties during the developmentally regulated maturation of neuromuscular synapses in both mammalian and Xenopus systems.

\section{Regulation of AChR trafficking and clustering at developing NMJs}

The presence of a high density of AChR clusters at postsynaptic membrane is the most prominent feature of the NMJs. Even prior to nerve innervation, AChRs are expressed and assembled in spontaneous clusters, called aneural AChR clusters, in Xenopus muscle cultures and other muscle cell lines [5, 17] (Figure 1b). During neuromuscular synaptogenesis, it is widely believed that the induction, assembly, and maturation of AChR clusters at postsynaptic site are initiated by the stimulation of a nerve-derived heparan-sulfate proteoglycan agrin (AGRN) [18]. Binding of agrin to LRP4 activates MuSK that consequently causes AChR clustering through a cytoplasmic linker protein rapsyn, receptor associated protein of the synapse (RAPSN) [19-21]. In addition, agrin-induced MuSK activation causes dephosphorylation of aneural AChR clusters by tyrosine phosphatases, which eventually leads to the dispersal of aneural AChR clusters [22]. There are two non- 
mutually exclusive hypotheses that have been proposed to mediate redistribution of AChRs from extra-synaptic regions to the nascent postsynaptic sites for clustering. First, the diffusion-trap mechanism suggests that nerve-induced assembly of cytoskeletal scaffolds may act as a trap to recruit and/or stabilize the mobile AChR molecules that exhibit random Brownian motion in the muscle membrane surface [23]. Second, AChRs at the aneural cluster may be internalized, transported via the endocytic vesicles, and then re-inserted to the synaptic sites by the vesicular trafficking mechanism. The recycling of internalized AChRs is a calcium-dependent process, mediated by calcium-activated calmodulin-dependent kinase II (CaMKII) activity [24].

Both actin and microtubule cytoskeleton are shown to be crucial for vesicular trafficking and clustering of AChRs at the postsynaptic sites [25, 26]. Agrin activates a small GTPase Cdc42 that in turn regulates Arp2/3 complex to promote actin polymerization through the activation of Wiskott Aldrich Syndrome protein (WASp) [27]. The postsynaptic stabilization of AChR clusters is achieved by the rapsyn-mediated linkage of the AChR to the cytoskeleton-anchored dystrophin-glycoprotein complex [28]. Apart from serving as a stable cytoskeletal scaffold for the docking and anchoring of structural and signaling molecules, our previous studies have provided definitive evidence to show that another dynamic pool of Factin, regulated by actin depolymerizing factor (ADF)/cofilin, actively facilitates the sitedirected delivery of AChRs to the nascent postsynaptic sites by modulating dynamic actin turnover locally. ADF/cofilin may break the cortical actin barrier for allowing the fusion of AChR-containing vesicles with the plasma membrane [5]. As for the involvement of microtubules for AChR clustering, a recent study has demonstrated that an increase in microtubule capturing leads to an increase in AChR density at the postsynaptic membrane [29]. Agrin inhibits the phosphorylation of serine residues of microtubule plus end-tracking protein CLASP2. Phosphatidylinositol-3,4,5-triphosphate ( $\left.\mathrm{PIP}_{3}\right)$-binding protein LL5 $\beta$ promotes the binding of CLASP 2 to microtubule plus-ends in regulating microtubule capturing.

\section{Pathogenic mechanisms of AChR endocytosis and degradation in MG}

MG is a prototypical autoimmune neuromuscular disease caused by the attack of autoAbs against some crucial postsynaptic proteins at NMJs. It has been shown from the clinical data that around $85 \% \mathrm{MG}$ patients contain autoAbs against complex epitopes in different subunits of AChR, including cholinergic receptor nicotinic alpha 1 (CHRNA1), 
cholinergic receptor nicotinic beta 1 (CHRNB1), cholinergic receptor nicotinic delta (CHRND), cholinergic receptor nicotinic epsilon (CHRNE), and cholinergic receptor nicotinic gamma (CHRNG) (Table 1), which represent the most typical type of autoAbs in seropositive MG (SPMG) patients [30]. The pathogenic mechanisms underlying SPMG are relatively well characterized, including antigenic modulation, activation of complement system, and inhibition of AChR channel function [7•, 31•] (Figure 2). The antigenic modulation is initiated by the binding of autoAbs to antigens in AChR proteins, which induces receptor crosslinking and internalization, and eventually leads to the loss of surface AChRs from the postsynaptic membrane (Figure 2a). The reduced number of postsynaptic AChRs decreases the amplitude of nerve-evoked end-plate potentials, and thereby the nerve signal is unlikely to initiate muscle contraction by depolarizing the muscle membrane potential beyond the threshold for generating an action potential. In addition, the direct binding of autoAbs occupies the ligand-binding sites of AChRs, which prevents the activation of AChR from binding with ACh ligands released from the nerve terminal and leads to the inhibition of channel function (Figure $2 \mathrm{~b}$ ). Regarding the activation of complement system, autoAbs against AChR belong to IgG1 subclass, consisting of bivalent Fc (fragment crystallizable region). This suggests that binding of IgG1 to AChR activates the classical complement system. Assembly of the membrane attack complex (MAC) triggers the influx of calcium ions that causes the destruction of postsynaptic membrane and junctional folds, as well as the lysis of muscle fibers (Figure 2c). Taken together, antigenic modulation, complement activation, and channel inhibition are the major pathological mechanisms of MG.

The remaining $15 \% \mathrm{MG}$ patients that do not have anti-AChR autoAbs were initially considered as seronegative MG (SNMG) patients [30, 32, 33]; however, a considerable amount of work has been carried out to search for novel antigenic reactivity in SNMG patients in the past decades. Now, more than half of the previously identified SNMG patients indeed pose autoAbs against MuSK. Unlike MG caused by autoAbs against AChR (AChRMG), the pathogenic mechanism of autoAbs against MuSK (MuSK-MG) is not well understood. It is believed that the pathogenic subclass of autoAbs involved in MuSK-MG belongs to IgG4 [34]. The presence of monovalent Fc due to Fab (fragment antigen-binding region) arm exchange making MuSK IgG4 follows a different immunological pathway to AChR depletion as in AChR-MG. This evidence suggests that the depletion of AChR caused by anti-MuSK autoAbs is not complement-related. It has been demonstrated that when the 
passive transfer of the MG patient-derived IgG with high titres of MuSK antibody into mice, there is an internalization of MuSK followed by a reduction in the density and cluster size of AChRs at NMJs [32]. It is possible that the dimerization-induced MuSK activation by autoAbs leads to its depletion. However, the exact pathogenic mechanism is yet to be revealed. Apart from the autoAbs against MuSK, there is a growing list of autoAbs has been identified in those initially identified SNMG patients, which attack other synaptic proteins at the NMJs [7•], including AGRN, LRP4, RAPSN, collagen like tail subunit of asymmetric acetylcholinesterase (COLQ), cortactin (CTTN), potassium voltage-gated channel subfamily A member 4 (KCNA4), ryanodine receptor 1 (RYR1), and titin (TTN) (Table 1). With the identification of these novel autoAbs, the percentage of SNMG has now been dramatically reduced. Considering the high sequence homology of all these proteins between Xenopus and human (as summarized in Table 1), Xenopus tissue cultures offer an ideal assay for the study of autoAb-mediated pathogenic mechanisms underlying AChR endocytosis, trafficking, and degradation in MG pathogenesis.

\section{Xenopus cultures as an alternative cell-based assay for MG diagnosis}

In 1976, Lindstrom's group firstly described a serological assay for MG diagnosis by detecting the presence of anti-AChR autoAbs with radioimmunoprecipitation assay (RIPA) [35]. In short, radioactive $\alpha$-BTX molecules are added to bind with AChR proteins in human muscle extracts that serve as antigens for autoAbs. After incubating the patient's serum containing potential pathogenic autoAbs, co-precipitation is performed with the anti-human IgG antibodies. Radioactivity of the pellets indicates the presence of anti-AChR autoAbs in the patient serum sample. An alternative approach is enzyme-linked immunosorbent assay (ELISA), which was first adopted for MG diagnosis in 1984 [36]. In this assay, recombinant AChR proteins are coated on flat-bottom microtiter plate, followed by an incubation of patient serum samples. Color given out by the reaction between peroxidase-conjugated antihuman IgG and the substrate indicates the presence of autoAbs against AChR in the serum sample. As the recombinant AChR proteins are in solution phase, some autoAbs against clustered AChR may not be detected in RIPA [37]. Besides, insufficient blocking may also give false positive results in ELISA.

Despite RIPA and commercial ELISA kits have been widely adopted for MG diagnosis, their sensitivity is questionable. A more sensitive approach using the cell-based assay (CBA) is therefore developed. It has been shown that $66 \%$ of serum samples from 
previously identified SNMG patients indeed contain low-affinity AChR-binding IgG against clustered AChR when CBA was used as the serological test [38・•]. Co-expression of complementary DNA sequences encoding $\mathrm{AChR}$ and the anchoring protein, rapsyn, in human embryonic kidney (HEK) cells mimics the postsynaptic specializations of NMJ in vivo where AChRs are highly concentrated. The presence of autoAbs in the patient serum sample is then determined by immunocytochemistry. In comparison to RIPA and ELISA, CBA is considered as a more reliable approach for MG diagnosis [38••], which allows the expression of AChR proteins in a cellular environment to enable the localization and clustering of AChRs at a high density in anchorage to membrane surface via rapsyn. This apparently improves the sensitivity of detecting the binding of low reactivity autoAbs against clustered AChRs.

Apart from using heterologous cell lines, our recent study described the use of Xenopus tissue cultures as an alternative CBA for MG diagnosis and research [39••]. We were able to use Xenopus tissue cultures to detect the pathogenicity of serum samples from both SPMG and SNMG patients, as evidenced by a significant reduction of aneural and nerve-induced AChR clusters after incubating with MG patient serum samples (Figure 1b). The interaction between autoAbs in the serum samples from MG patients and proteins in the postsynaptic apparatus would cause the endocytosis and disassembly of aneural and nerveinduced AChR clusters in pure muscle cultures and nerve-muscle co-cultures, respectively. Apart from the cell imaging approach to quantify the effects of MG serum on AChR cluster dispersal, Xenopus neuron-myoball co-cultures can also be employed to test the effects of MG patient serum samples on synaptic functions of NMJs by in vitro electrophysiological recordings (Figure 1c). Spontaneous synaptic currents (SSCs) can be measured by the whole-cell patch-clamp recording method on 1-day-old neuron-myoball co-cultures before and after the incubation of MG patient serum samples. The change in SSC frequency and/or amplitude implicates the effects of MG serum on presynaptic and/or postsynaptic inhibitions of neuromuscular functions, respectively. Hence, the possibility of assessing the structural (cell imaging approach) and functional (electrophysiology approach) defects of NMJs after the treatment of pathogenic MG serum samples in Xenopus tissue cultures infers their potential to be used as an alternative CBA in MG diagnostic practice.

One might argue on the feasibility of translating the knowledge obtained from the Xenopus model into our understanding of human diseases. As shown in the recent completion of sequencing data, Xenopus genome shares a high level of spatial and functional 
collinearity of genes with humans [40]. Xenopus is well positioned between distant invertebrates, like the fruit fly (Drosophila) and the worm (C. elegans), and the less accessible mammalian models [41]. Importantly, we have found that the amino acid sequence of some postsynaptic proteins in association to the MG pathogenesis shows a high degree of similarities between Xenopus and human (Table 1). For instance, the amino acid sequences of AChR $\alpha 1$ subunit, CHRNA1, the ACh-binding subunit of AChR, in Xenopus tropicalis and Xenopus laevis share $74 \%$ and $73 \%$ similarity to that in human, respectively. Another important postsynaptic protein, MuSK, also shows more than $80 \%$ sequence homology between Xenopus species and human. Taken together, this gives a strong argument that Xenopus tissue culture is an ideal platform for the serological diagnosis and the pathological research of MG.

\section{In search of therapeutic strategies for the treatment of MG using Xenopus tissue cultures}

Many potential strategies have been identified to alleviate the symptoms of muscle weakness after the onset of MG [7•]. One of the most commonly used medications is the reversible acetylcholinesterase (AChE) inhibitor, pyridostigmine [42]. The increased level and duration of neurotransmitter acetylcholine by inhibiting AChE activity at the synaptic cleft can alleviate muscle weakness in MG patients. However, the transient and mild effects of AChE inhibition are insufficient to treat MG [43], and may also contribute to various side effects, such as muscarinic overactivity, nicotinic side effects, and cholinergic crisis state [44]. Other common treatments to MG also include the immunosuppressant drug, the plasmapheresis, the immunoglobulin therapy, and thymectomy [7•]. These treatment strategies, however, cannot give a complete remission for MG patients, which urge the development of advanced therapeutic approaches for MG treatment in the future.

At mature NMJs, it has long been believed that AChRs are metabolically stable in the postsynaptic membrane at the mature NMJs, until they are internalized and targeted to lysosomes for protein degradation [45]. In fact, some internalized AChRs are recycled and inserted back to the postsynaptic membrane for maintaining a high postsynaptic AChR density $[24,46]$. Using biotin- $\alpha$-BTX and streptavidin-conjugates of different fluorophores, it is possible to distinguish various pools of AChRs, namely pre-existing, recycling, and newly synthesized AChRs [46, 47]. This sequential labeling approach will allow us to investigate the trafficking mechanisms of endocytosed AChR vesicles following endocytosis. 
Our long-term goal is to develop a potential therapeutic strategy that alleviates the loss of AChRs at NMJs in MG pathogenesis. One of the possible therapeutic approaches is to manipulate the trafficking and stability of synaptic AChR clusters. Given the fact that the loss of AChR clusters is triggered by autoAb-induced crosslinking followed by endocytosis of AChRs, a therapeutic strategy can be developed to modulate the rate of $\mathrm{AChR}$ endocytosis and/or to regulate the recycling of endocytosed AChRs, so as to maintain the high density of AChR clusters for efficient neurotransmission between motor neurons and muscle fibers. Our recent studies using Xenopus muscle cells have demonstrated the spatial and temporal correlation between $\mathrm{ADF} /$ cofilin localization and the clustering of AChRs at developing NMJs [5]. We also observed the disassembly of AChR clusters and the disappearance of $\mathrm{ADF} /$ cofilin localization upon the treatment of $\mathrm{MG}$ patient serum samples, suggesting that pathogenic autoAbs regulate $\mathrm{ADF} /$ cofilin localization and function for the dispersal of $\mathrm{AChR}$ clusters [39••]. It is of interest to further investigate if manipulating the activity of $\mathrm{ADF} /$ cofilin or its upstream regulators could inhibit the endocytosis and degradation of AChR clusters in response to MG serum treatment.

Our recent study reported a procedure to mimic the pathogenic autoAbs for inducing the crosslinking and endocytosis of AChRs in Xenopus tissue cultures [48•]. In that study, we took advantage of the multivalent interaction between biotin and streptavidin to crosslink the biotin- $\alpha$-BTX-labeled AChR molecules by streptavidin-quantum dots (QDs) (Figure 2d). QDs are nanometer-sized semiconductor particles that are much brighter and more stable than traditional organic fluorescent probes, which allow us to visualize and perform longterm tracking of internalized AChR vesicles upon receptor crosslinking-induced AChR endocytosis. The unique features of QDs, which include the direct relationship between the QD-emitted wavelength of fluorescence emission and its size, the multicolor imaging from wide excitation and narrow emission range, and the possible detection of QDs in transmission electron microscopy (TEM) without chemical staining [49], offer a powerful tool for singlemolecule imaging and tracking. By using correlative light and electron microscopy (CLEM) [50], high resolution correlative microscopy images can be acquired to characterize the particular subcellular structures in association with the endocytic trafficking and lysosomal degradation pathways of endocytosed AChRs upon the crosslinking action by streptavidinQDs. Therefore, QDs can be employed not only to visualize and track the surface movement of AChRs during the formation of synaptic AChR clusters in live muscle cells [51], but also the multivalent interaction between biotin and streptavidin allows us to track the internalized 
AChR vesicles following the crosslinking-induced endocytosis [48•]. As this crosslinking effect mimics the antigenic modulation, one of the pathogenic mechanisms of autoAbs that induces internalization of crosslinked AChR clusters in MG patients, a possible treatment may be developed to interfere the clathrin-mediated endocytic pathway, which has been implicated to regulate the crosslinking-induced AChR endocytosis in cultured muscle cells [48•]. Therefore, the reduced level of antigenic modulation should prevent the loss of AChR clusters in postsynaptic membrane. Taken together, Xenopus tissue cultures offer a simple in vitro assay to study and manipulate the pathogenic mechanisms in MG.

\section{Conclusion}

In light of the potential pitfalls of all current diagnostic assays described above, it is essential to develop a more reliable, sensitive, and specific tool for the diagnosis of $\mathrm{MG}$, even at the early stage of pathogenesis. Although most CBAs are relatively labor intensive, Xenopus tissue culture-based serological test is a promising tool for the initial clinical diagnosis of MG by testing autoAb pathogenicity in vitro. This assay can also be employed to distinguish the disease severity among different patients, and to monitor the course of the disease and the efficacy of treatment received for a particular MG patient. Additionally, Xenopus tissue cultures provide an important research platform for elucidating the pathogenic mechanisms underlying AChR endocytosis in both seropositive and seronegative MG patients. Therefore, results of these studies using Xenopus tissue cultures should contribute to a better understanding of the etiology of $\mathrm{MG}$ and provide a therapeutic intervention for this malady in the future.

\section{Acknowledgements}

This work is partly supported by the Health and Medical Research Fund (HMRF, Project No. 04151086) from Food and Health Bureau (FHB) in Hong Kong. 


\section{Figures}

(a)
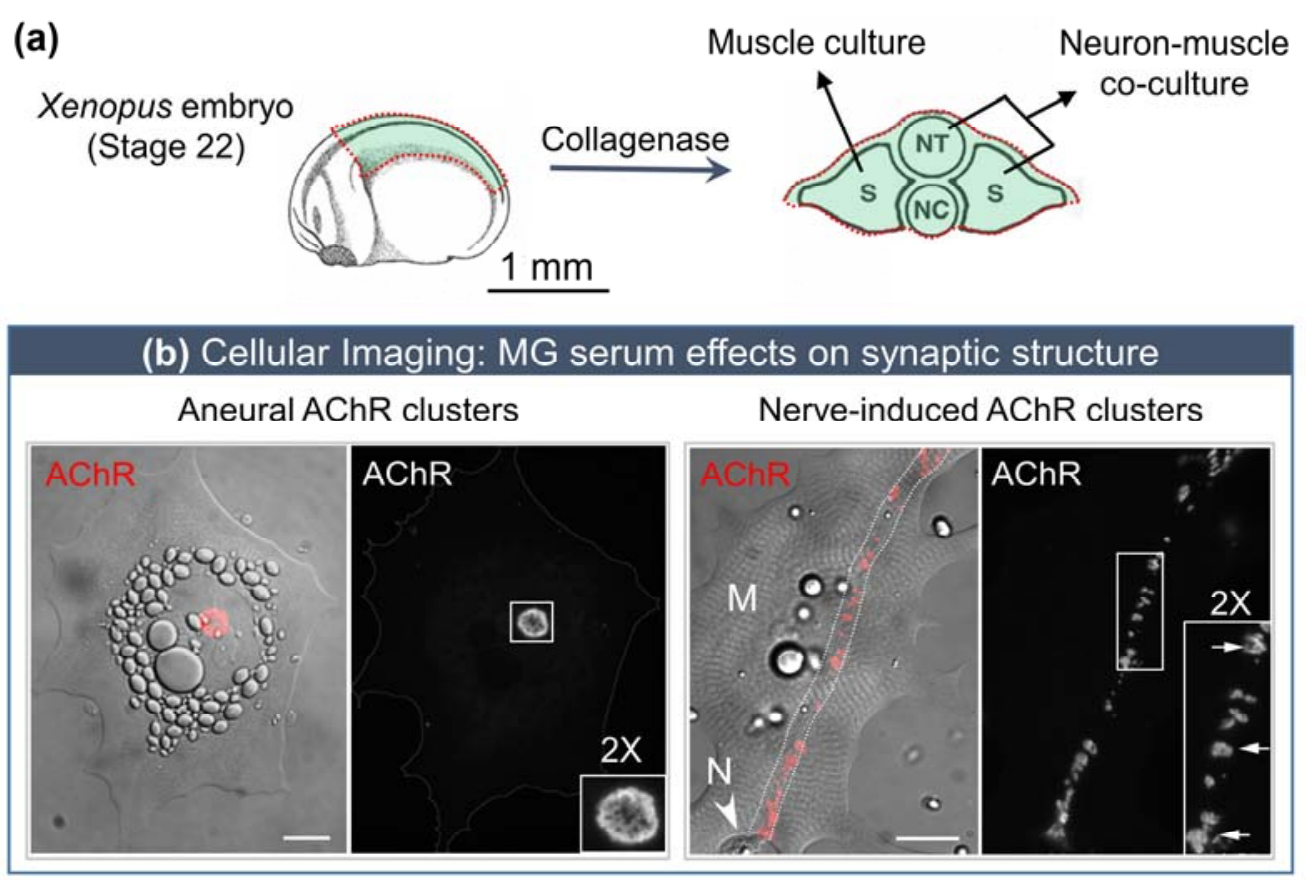

(c) Electrophysiology: MG serum effects on synaptic function

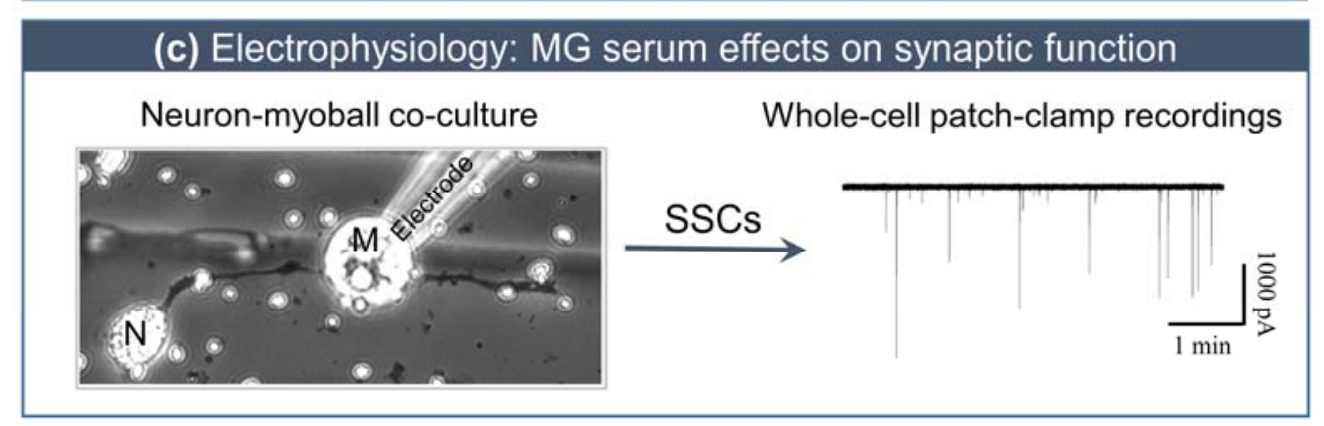

Figure 1. Experimental approaches to examine the effects of autoantibodies in MG patient serum on the synaptic structure and function of NMJs in vitro. (a) The dorsal part (dotted lines, highlighted regions in green) of a 1-day-old Xenopus embryo (at stage 22), which contains neural tube (NT), somite (S), and notochord (NC), is dissected and then subjected to enzymatic collagenase treatment. Somite tissues are dissociated and plated separately to prepare muscle cultures, or together with neural tube tissues to prepare neuron-muscle co-cultures. (b) Left: Spontaneously formed aneural AChR clusters (inset) are observed by live labeling the muscle cells with rhodamineconjugated $\alpha$-bungarotoxin (red). Right: In neuron-muscle co-cultures, nerve-induced AChR clusters (inset, arrows) are found to be spatially localized along the contact sites between neurons and muscle cells (dotted lines). Arrowhead points to the cell body of a spinal neuron. (c) Dissociated muscle cells (M) and spinal neurons (N) are plated on plain glass coverslips for electrophysiological recordings. Spontaneous synaptic currents (SSCs) are recorded from the innervated muscle cells in 1day-old neuron-myoball co-cultures by the whole-cell patch clamp technique. The effects of MG patient serum samples on the synaptic structure and function of NMJs can be examined by cellular imaging and electrophysiology approaches, respectively. Scale bars represent $20 \mu \mathrm{m}$. 


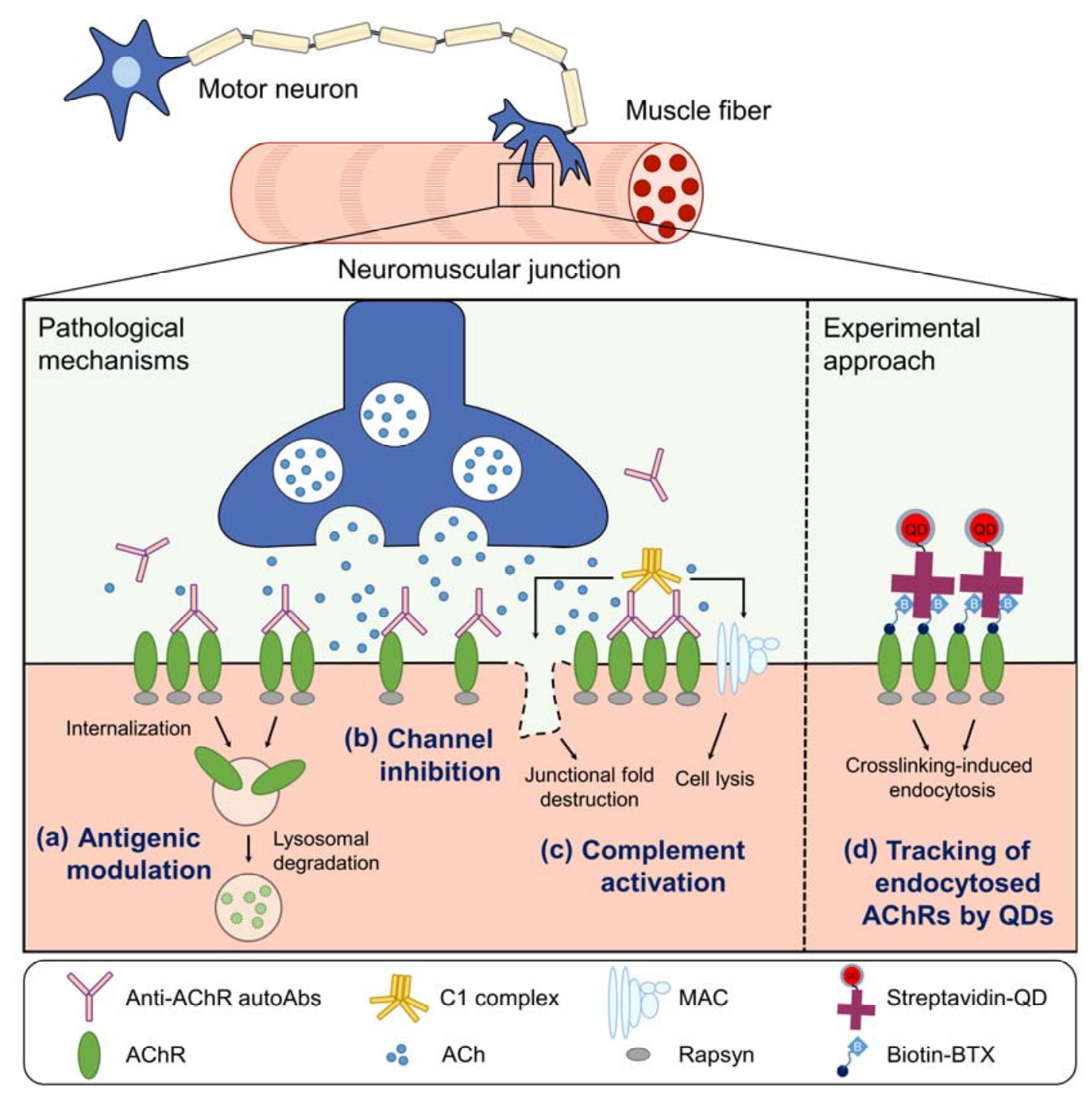

Figure 2. The pathological mechanisms of anti-AChR autoAbs and the experimental approach to mimic the pathogenic autoAbs for inducing the crosslinking and internalization of AChRs in

MG. Pathological mechanisms: (a) Antigenic modulation: The binding of autoAbs to AChRs causes crosslinking and internalization of AChRs. Then, the endocytosed AChRs are targeted to lysosomal degradation, leading to the loss of AChR density at the end plates of NMJs. (b) Channel inhibition: The binding of autoAbs to the ligand-binding site of AChRs directly inhibits the function of AChR channels in mediating membrane depolarization. (c) Complement activation: The binding of autoAbs to AChR leads to the assembly of $\mathrm{C} 1$ complement complex and membrane attack complex (MAC), which consequently causes the destruction of junctional fold structures in the sarcolemma and the cell lysis through the calcium influx via MAC. Experimental approach: (d) Tracking of endocytosis AChRs by QDs: To mimic the crosslinking-induced endocytosis mediated by autoAbs, AChRs are first labeled with biotin-conjugated $\alpha$-bungarotoxin (biotin-BTX), followed by the addition of streptavidin-conjugated quantum dot (streptavidin-QD). Due to the multivalent interaction between biotin and streptavidin, QD allows us to visualize and perform long-term tracking of AChR vesicles upon receptor crosslinking-induced endocytosis in live cells. 


\section{References}

1. Danilchick M, Peng HB, Kay BK. Xenopus laevis: Practical uses in cell and molecular biology. Pictorial collage of embryonic stages. Methods Cell Biol. 1991;36:679-81.

2. Beck CW, Slack JM. An amphibian with ambition: a new role for Xenopus in the 21st century. Genome Biol. 2001;2(10):REVIEWS1029.

3. Peng HB, Baker LP, Chen Q. Tissue culture of Xenopus neurons and muscle cells as a model for studying synaptic induction. Methods Cell Biol. 1991;36:511-26.

4. Cohen MW. Development of an amphibian neuromuscular junction in vivo and in culture. J Exp Biol. 1980;89:43-56.

5. Lee CW, Han J, Bamburg JR, et al. Regulation of acetylcholine receptor clustering by ADF/cofilindirected vesicular trafficking. Nat Neurosci. 2009;12(7):848-56. doi:10.1038/nn.2322.

6. Proszynski TJ, Gingras J, Valdez G, et al. Podosomes are present in a postsynaptic apparatus and participate in its maturation. Proc Natl Acad Sci U S A. 2009;106(43):18373-8. doi:10.1073/pnas.0910391106.

7. - Gilhus NE, Skeie GO, Romi F, et al. Myasthenia gravis - autoantibody characteristics and their implications for therapy. Nat Rev Neurol. 2016;12(5):259-68. doi:10.1038/nrneurol.2016.44.

This paper provides an extensive review on the pathophysiology of different autoantibodies in MG, and on different therapeutic approaches to MG treatment.

8. Lindstrom JM. Acetylcholine receptors and myasthenia. Muscle \& Nerve. 2000;23(4):453-77. doi:10.1002/(SICI)1097-4598(200004)23:4<453::AID-MUS3>3.0.CO;2-O.

9. Higuchi O, Hamuro J, Motomura M, et al. Autoantibodies to low-density lipoprotein receptorrelated protein 4 in myasthenia gravis. Ann Neurol. 2011;69(2):418-22. doi:10.1002/ana.22312.

10. Hoch W, McConville J, Helms S, et al. Auto-antibodies to the receptor tyrosine kinase MuSK in patients with myasthenia gravis without acetylcholine receptor antibodies. Nat Med. 2001;7(3):365-8. doi:10.1038/85520.

11. Sanes JR, Lichtman JW. Development of the vertebrate neuromuscular junction. Annu Rev Neurosci. 1999;22:389-442. doi:10.1146/annurev.neuro.22.1.389.

12. Harrison RG. Observations on the living developing nerve fiber. Anat Rec. 1907:116-28.

13. Anderson MJ, Cohen MW, Zorychta E. Effects of innervation on the distribution of acetylcholine receptors on cultured muscle cells. J Physiol. 1977;268(3):731-56.

14. Anderson MJ, Cohen MW. Nerve-induced and spontaneous redistribution of acetylcholine receptors on cultured muscle cells. J Physiol. 1977;268(3):757-73.

15. Xie ZP, Poo MM. Initial events in the formation of neuromuscular synapse: rapid induction of acetylcholine release from embryonic neuron. Proc Natl Acad Sci U S A. 1986;83(18):7069-73.

16. Missias AC, Chu GC, Klocke BJ, et al. Maturation of the acetylcholine receptor in skeletal muscle: regulation of the AChR gamma-to-epsilon switch. Dev Biol. 1996;179(1):223-38. doi:10.1006/dbio.1996.0253.

17. Kummer TT, Misgeld T, Lichtman JW, et al. Nerve-independent formation of a topologically complex postsynaptic apparatus. J Cell Biol. 2004;164(7):1077-87. doi:10.1083/jcb.200401115.

18. Sanes JR, Lichtman JW. Induction, assembly, maturation and maintenance of a postsynaptic apparatus. Nat Rev Neurosci. 2001;2(11):791-805. doi:10.1038/35097557.

19. Kim N, Stiegler AL, Cameron TO, et al. Lrp4 is a receptor for Agrin and forms a complex with MuSK. Cell. 2008;135(2):334-42. doi:10.1016/j.cell.2008.10.002.

20. Zhang B, Luo S, Wang Q, et al. LRP4 serves as a coreceptor of agrin. Neuron. 2008;60(2):285-97. doi:10.1016/j.neuron.2008.10.006. 
21. Choi KR, Berrera M, Reischl M, et al. Rapsyn mediates subsynaptic anchoring of PKA type I and stabilisation of acetylcholine receptor in vivo. J Cell Sci. 2012;125(Pt 3):714-23.

doi:10.1242/jcs.092361.

22. Dai Z, Peng HB. A role of tyrosine phosphatase in acetylcholine receptor cluster dispersal and formation. J Cell Biol. 1998;141(7):1613-24.

23. Geng L, Qian YK, Madhavan R, et al. Transmembrane mechanisms in the assembly of the postsynaptic apparatus at the neuromuscular junction. Chem Biol Interact. 2008;175(1-3):108-12. doi:10.1016/j.cbi.2008.04.017.

24. Martinez-Pena y Valenzuela I, Mouslim C, Akaaboune M. Calcium/calmodulin kinase IIdependent acetylcholine receptor cycling at the mammalian neuromuscular junction in vivo. $\mathrm{J}$ Neurosci. 2010;30(37):12455-65. doi:10.1523/JNEUROSCI.3309-10.2010.

25. Connolly JA, Oldfin BV. Microtubules and the formation of acetylcholine receptor clusters in chick embryonic muscle cells. Eur J Cell Biol. 1985;39(1):173-8.

26. Dai Z, Luo X, Xie H, et al. The actin-driven movement and formation of acetylcholine receptor clusters. J Cell Biol. 2000;150(6):1321-34.

27. Cartaud A, Stetzkowski-Marden F, Maoui A, et al. Agrin triggers the clustering of raft-associated acetylcholine receptors through actin cytoskeleton reorganization. Biol Cell. 2011;103(6):287-301. doi:10.1042/BC20110018.

28. Apel ED, Roberds SL, Campbell KP, et al. Rapsyn may function as a link between the acetylcholine receptor and the agrin-binding dystrophin-associated glycoprotein complex. Neuron. 1995;15(1):115-26.

29. Basu S, Sladecek S, Martinez de la Pena y Valenzuela I, et al. CLASP2-dependent microtubule capture at the neuromuscular junction membrane requires LL5beta and actin for focal delivery of acetylcholine receptor vesicles. Mol Biol Cell. 2015;26(5):938-51. doi:10.1091/mbc.E14-06-1158.

30. Leite MI, Jacob S, Viegas S, et al. IgG1 antibodies to acetylcholine receptors in 'seronegative' myasthenia gravis. Brain. 2008;131(Pt 7):1940-52. doi:10.1093/brain/awn092.

31. Phillips WD, Vincent A. Pathogenesis of myasthenia gravis: update on disease types, models, and mechanisms. F1000Res. 2016;5. doi:10.12688/f1000research.8206.1.

This review paper evaluates the clinical conditions and pathogenic mechanisms of different subsets of MG patients in relationship to their distinct target specificities and isotypes of autoantibodies.

32. Cole RN, Ghazanfari N, Ngo ST, et al. Patient autoantibodies deplete postsynaptic musclespecific kinase leading to disassembly of the ACh receptor scaffold and myasthenia gravis in mice. $\mathrm{J}$ Physiol. 2010;588(Pt 17):3217-29. doi:10.1113/jphysiol.2010.190298.

33. Klooster R, Plomp JJ, Huijbers MG, et al. Muscle-specific kinase myasthenia gravis IgG4 autoantibodies cause severe neuromuscular junction dysfunction in mice. Brain. 2012;135( $\mathrm{Pt} 4): 1081$ 101. doi:10.1093/brain/aws025.

34. Koneczny I, Cossins J, Waters P, et al. MuSK myasthenia gravis IgG4 disrupts the interaction of LRP4 with MuSK but both IgG4 and IgG1-3 can disperse preformed agrin-independent AChR clusters. PLoS One. 2013;8(11):e80695. doi:10.1371/journal.pone.0080695.

35. Lindstrom JM, Seybold ME, Lennon VA, et al. Antibody to acetylcholine receptor in myasthenia gravis. Prevalence, clinical correlates, and diagnostic value. Neurology. 1976;26(11):1054-9.

36. Kawanami S, Tsuji R, Oda K. Enzyme-linked immunosorbent assay for antibody against the nicotinic acetylcholine receptor in human myasthenia gravis. Ann Neurol. 1984;15(2):195-200. doi:10.1002/ana.410150214.

37. Rodriguez Cruz PM, Al-Hajjar M, Huda S, et al. Clinical Features and Diagnostic Usefulness of Antibodies to Clustered Acetylcholine Receptors in the Diagnosis of Seronegative Myasthenia Gravis. JAMA Neurol. 2015;72(6):642-9. doi:10.1001/jamaneurol.2015.0203. 
38. • Rodriguez Cruz PM, Huda S, Lopez-Ruiz P, et al. Use of cell-based assays in myasthenia gravis and other antibody-mediated diseases. Exp Neurol. 2015;270:66-71.

doi:10.1016/j.expneurol.2015.01.011.

This paper reviews the principles of CBA to be used for MG diagnosis and highlights its advantages over other diagnostic assays.

39. •• Yeo HL, Lim JY, Fukami Y, et al. Using Xenopus tissue cultures for the study of myasthenia gravis pathogenesis. Dev Biol. 2015;408(2):244-51. doi:10.1016/j.ydbio.2015.02.017.

This is the first study to describe the use of Xenopus tissue cultures for screening the pathogenicity of serum samples from seropositive and seronegative MG patients.

40. Hellsten U, Harland RM, Gilchrist MJ, et al. The genome of the Western clawed frog Xenopus tropicalis. Science. 2010;328(5978):633-6. doi:10.1126/science.1183670.

41. Wheeler GN, Brandli AW. Simple vertebrate models for chemical genetics and drug discovery screens: lessons from zebrafish and Xenopus. Dev Dyn. 2009;238(6):1287-308. doi:10.1002/dvdy.21967.

42. Schwab RS, Timberlake WH. Pyridostigmin (mestinon) in the treatment of myasthenia gravis. N Engl J Med. 1954;251(7):271-2. doi:10.1056/NEJM195408122510706.

43. Saperstein DS, Barohn RJ. Management of myasthenia gravis. Semin Neurol. 2004;24(1):41-8. doi:10.1055/s-2004-829586.

44. Gehi A, Benatar M, Langberg J. Treatment of pyridostigmine-induced AV block with hyoscyamine in a patient with myasthenia gravis. J Cardiovasc Electrophysiol. 2008;19(2):214-6. doi:10.1111/j.1540-8167.2007.00938.x.

45. Gardner JM, Fambrough DM. Acetylcholine receptor degradation measured by density labeling: effects of cholinergic ligands and evidence against recycling. Cell. 1979;16(3):661-74.

46. Bruneau E, Sutter D, Hume RI, et al. Identification of nicotinic acetylcholine receptor recycling and its role in maintaining receptor density at the neuromuscular junction in vivo. J Neurosci. 2005;25(43):9949-59. doi:10.1523/JNEUROSCI.3169-05.2005.

47. Bruneau EG, Akaaboune M. The dynamics of recycled acetylcholine receptors at the neuromuscular junction in vivo. Development. 2006;133(22):4485-93. doi:10.1242/dev.02619.

48. - Lee CW, Zhang H, Geng L, et al. Crosslinking-induced endocytosis of acetylcholine receptors by quantum dots. PLoS One. 2014;9(2):e90187. doi:10.1371/journal.pone.0090187.

This study takes advantage of the unique features of $Q D$ s and the multivalency of biotinstreptavidin interaction to mimic the crosslinking-induced AChR endocytosis in MG pathogenesis.

49. Ben NGG, Thomas JD, Benjamin LS, et al. Correlated light and electron microscopic imaging of multiple endogenous proteins using Quantum dots. Nature Methods. 2005;2(10):743.

doi:10.1038/nmeth791.

50. Giepmans BN, Deerinck TJ, Smarr BL, et al. Correlated light and electron microscopic imaging of multiple endogenous proteins using Quantum dots. Nat Methods. 2005;2(10):743-9.

doi:10.1038/nmeth791.

51. Geng L, Zhang HL, Peng HB. The formation of acetylcholine receptor clusters visualized with quantum dots. BMC Neurosci. 2009;10:80. doi:10.1186/1471-2202-10-80. 